\title{
Doubly-Generalized LDPC Codes: Stability Bound over the BEC
}

Enrico Paolini, Member, IEEE, and

Marc Fossorier, Fellow, IEEE, and

Marco Chiani, Senior Member, IEEE.

\author{
Corresponding Address: \\ Marco Chiani \\ DEIS, University of Bologna \\ V.le Risorgimento 2 \\ 40136 Bologna, ITALY
}

Tel: +39-051-2093084 Fax: +39-051-2093540

e-mail: marco.chiani@unibo.it 


\begin{abstract}
The iterative decoding threshold of low-density parity-check (LDPC) codes over the binary erasure channel (BEC) fulfills an upper bound depending only on the variable and check nodes with minimum distance 2. This bound is a consequence of the stability condition, and is here referred to as stability bound. In this paper, a stability bound over the BEC is developed for doubly-generalized LDPC codes, where the variable and the check nodes can be generic linear block codes, assuming maximum a posteriori erasure correction at each node. It is proved that in this generalized context as well the bound depends only on the variable and check component codes with minimum distance 2 . A condition is also developed, namely the derivative matching condition, under which the bound is achieved with equality.
\end{abstract}

\title{
I. INTRODUCTION
}

LDPC codes [1] have been intensively studied in the last decade due to their capability to approach the Shannon limit under iterative belief-propagation decoding. An LDPC code of length $N$ and dimension $K$ can be graphically represented as a bipartite graph, known as Tanner graph, with $N$ variable nodes (VNs) and $M \geq N-K$ check nodes (CNs) [2]. In the Tanner graph, the degree of either a $\mathrm{VN}$ or a $\mathrm{CN}$ is defined as the number of edges connected to it. A degree- $n$ VN of an LDPC code can be interpreted as a length- $n$ repetition code, i.e., as a $(n, 1)$ linear block code repeating $n$ times its only information bit towards the check node decoder (CND). Instead, a degree- $n \mathrm{CN}$ of an LDPC code can be interpreted as a length- $n$ single parity-check (SPC) code, i.e., as a $(n, n-1)$ linear block code.

An extension of the concept of LDPC code is represented by doubly-generalized LDPC (DGLDPC) codes [3], where the VNs and the CNs are allowed to be generic $(n, k)$ linear block codes instead of repetition and SPC codes, respectively. If only the CND is generalized while all the VNs are repetition codes, then the code is said a generalized LDPC (GLDPC) code, or a Tanner code [2].

In a D-GLDPC code the codes used as VNs and CNs are called component codes. In this work each component code is supposed to be a linear block code having a minimum distance $d_{\min } \geq 2$. The VNs and the CNs which are not repetition or SPC codes, are referred to as generalized nodes. The corresponding code structure is depicted in Fig. 1. An $(n, k)$ generalized VN is characterized by $n$ connections towards the CND; moreover, $k$ of the $N$ D-GLDPC encoded 


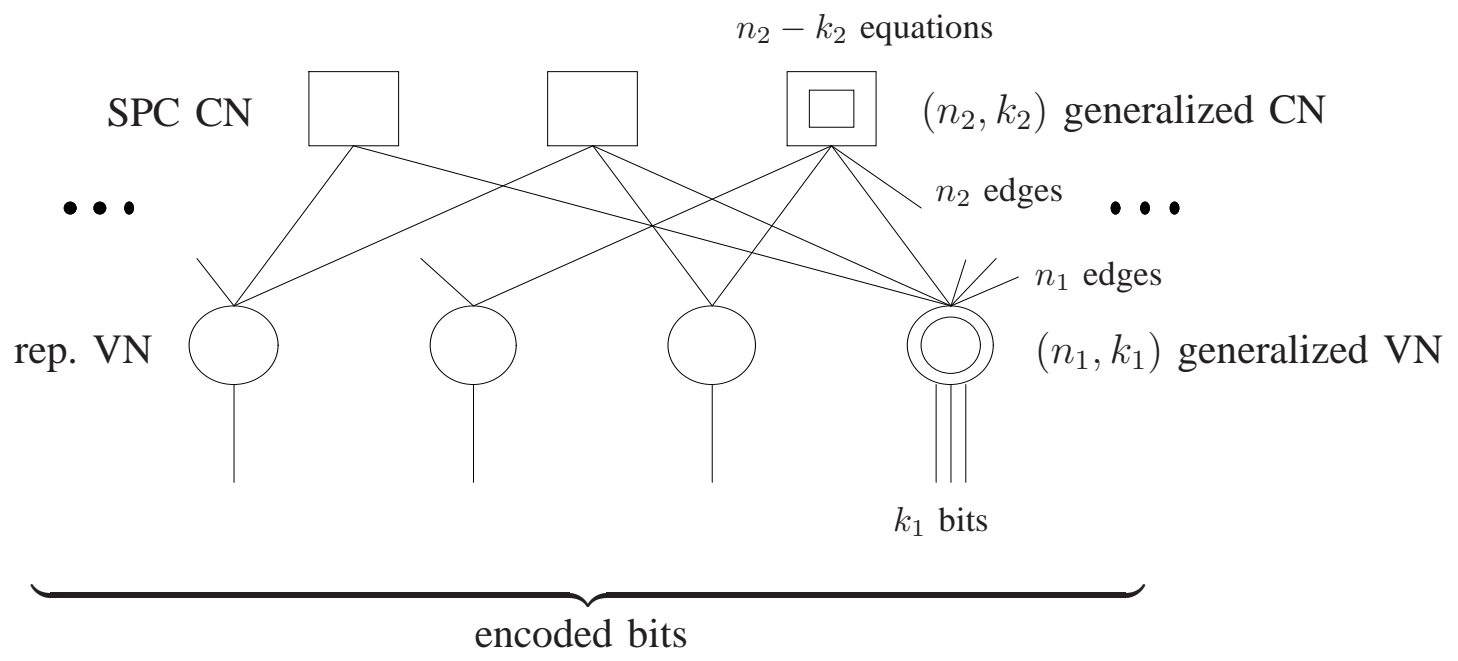

Fig. 1. Structure of a D-GLDPC code.

bits are associated with the $\mathrm{VN}$, and interpreted by the $\mathrm{VN}$ as its $k$ information bits. Then, the codeword length of a D-GLDPC code with $N_{V}$ VNs is $N=\sum_{i=1}^{N_{V}} k_{i}$ ( $k_{i}$ being the dimension of the $i$-th $\mathrm{VN})$. An $(n, k)$ generalized $\mathrm{CN}$ is characterized by $n$ connections towards the variable node decoder (VND), and is associated with $n-k$ independent parity check equations. Then, the number of parity-check equations for a D-GLDPC code with $N_{C}$ CNs is $M=\sum_{i=1}^{N_{C}}\left(n_{i}-k_{i}\right)\left(k_{i}\right.$ and $n_{i}$ being the dimension and length of the $i$-th check node, respectively). For a description of a D-GLDPC code iterative decoder over the AWGN channel and BEC we refer to [3] and [4], respectively.

For LDPC code ensembles, an important role is played by a theorem known as the stability condition [5]-[7]. The most important consequence of the stability condition is the possibility to upper bound the asymptotic iterative decoding threshold. If the communication channel is a BEC with erasure probability $q$, the stability condition leads to the following upper bound on the asymptotic threshold $q^{*}$ for the LDPC ensemble:

$$
q^{*} \leq\left[\lambda^{\prime}(0) \rho^{\prime}(1)\right]^{-1}
$$

The inequality (1) is referred to as stability bound in this papert. In (1), $\lambda^{\prime}(0)=\lambda_{2}$ is the fraction

\footnotetext{
${ }^{1}$ We use the nomenclature stability bound as, even though 11 is sometimes referred to as the stability condition, strictly speaking it is a consequence of it. For more details we refer to [7, Theorem 3.66] and the related discussion.
} 
of edges connected to the length-2 repetition VNs, while $\rho^{\prime}(1)$ is the derivative (computed at $x=1$ ) of the LDPC CNs degree distribution $\rho(x)=\sum_{j \geq 2} \rho_{j} x^{j-1}$, where $\rho_{j}$ is the fraction of edges connected to SPC CNs of length $j$. The bound (1) was first developed from density evolution. Next we propose a simple graphical interpretation of (1) using extrinsic information transfer (EXIT) charts [8]. Let us denote by $I_{A}$ the average a priori mutual information in input to the VND or to the CND. Furthermore, let us denote by $I_{E, V}\left(I_{A}, q\right)$ and $I_{E, C}\left(I_{A}\right)$ the average extrinsic information for the VND and CND respectively (these functions are usually referred to as EXIT functions). Then, (1) is equivalent to the following condition: for $q=q^{*}$, the derivative of the VND EXIT function $I_{E, V}\left(I_{A}, q\right)$, with respect to $I_{A}$ and evaluated at $I_{A}=1$, must be smaller than the derivative of the inverse CND EXIT function $I_{E, C}^{-1}\left(I_{A}\right)$ evaluated at $I_{A}=1$. That is (11) is equivalent to requiring

$$
\left.\frac{\partial I_{E, V}\left(I_{A}, q^{*}\right)}{\partial I_{A}}\right|_{I_{A}=1} \leq\left.\frac{\mathrm{d} I_{E, C}^{-1}\left(I_{A}\right)}{\mathrm{d} I_{A}}\right|_{I_{A}=1} .
$$

There exist LDPC degree distributions achieving the bound (1) with equality, so that their threshold over the BEC assumes the simple closed form $q^{*}=\left[\lambda^{\prime}(0) \rho^{\prime}(1)\right]^{-1}$. For such LDPC distributions, the first occurrence of a tangency point between the VND EXIT function $I_{E, V}\left(I_{A}, q\right)$ and the inverse CND EXIT function $I_{E, C}^{-1}\left(I_{A}\right)$ appears at $I_{A}=1$, i.e.

$$
\left\{\begin{array}{l}
I_{E, V}\left(1, q^{*}\right)=I_{E, C}^{-1}(1) \\
\left.\frac{\partial I_{E, V}\left(I_{A}, q^{*}\right)}{\partial I_{A}}\right|_{I_{A}=1}=\left.\frac{\mathrm{d} I_{E, C}^{-1}\left(I_{A}\right)}{\mathrm{d} I_{A}}\right|_{I_{A}=1}
\end{array}\right.
$$

For LDPC code ensembles characterized by VNs and CNs with degree at least 2, the first equality is always satisfied as both terms are equal to 1 . This occurs also for D-GLDPC codes with all variable and check component codes having a minimum distance $d_{\min } \geq 2$ [9], which is an assumption of this paper. Then, only the second equality is considered in the sequel, and is referred to as the derivative matching condition.

In this paper, the stability upper bound (1) and the derivative matching condition are extended to D-GLDPC codes (and to GLDPC codes as a sub-case). Our derivations lead to the conclusion that only the check and variable component codes with minimum distance $d_{\text {min }}=2$, including length-2 repetition codes and SPC codes, contribute to the stability bound. We also show that 
for D-GLDPC codes satisfying the derivative matching condition the asymptotic threshold over the BEC can be expressed by a simple formula.

The paper is organized as follows. Some definitions and the notation used in the paper are introduced in Section [II. In Section III the possibility to reduce the rank of a linear block code generator matrix by column elimination is discussed. Using these results, in Section IV and Section $\mathrm{V}$ the stability bound is developed for GLDPC codes and for D-GLDPC codes, respectively. Final remarks are given in Section VI,

\section{DEFINITIONS AND BASIC NOTATION}

We assume as transmission channel a BEC with erasure probability $q$. For a bipartite graph with random connections, the extrinsic channel (that is the channel over which the messages are exchanged between the VND and the CND during the iterative decoding process) is modelled as a second BEC with erasure probability $p$ depending on the decoding iteration [10], where it is readily proved that $I_{A}=1-p$. Since we express both the VND and the CND EXIT functions as functions of $p$ (and $q$ for the VND), their derivatives are evaluated at $p=0$ (corresponding to $I_{A}=1$ ). In this case (2) becomes

$$
\left.\frac{\partial I_{E, V}\left(p, q^{*}\right)}{\partial p}\right|_{p=0} \geq\left.\frac{\mathrm{d} I_{E, C}(p)^{-1}}{\mathrm{~d} p}\right|_{p=0} .
$$

Under the hypothesis of a random bipartite graph, the VND and CND EXIT functions can be expressed as

$$
I_{E, V}(p, q)=\sum_{i=1}^{\mathcal{I}_{V}} \lambda_{i} I_{E, V}^{(i)}(p, q)
$$

and

$$
I_{E, C}(p)=\sum_{i=1}^{\mathcal{I}_{C}} \rho_{i} I_{E, C}^{(i)}(p),
$$

respectively, where $\mathcal{I}_{V}$ and $\mathcal{I}_{C}$ are the number of different $\mathrm{VN}$ and $\mathrm{CN}$ types, $I_{E, V}^{(i)}(p, q)$ and $I_{E, C}^{(i)}(p)$ are the EXIT functions for the $i$-th VN type and for the $i$-th $\mathrm{CN}$ type, respectively, and $\lambda_{i}$ and $\rho_{i}$ are the fractions of edges towards the VNs of type $i$ and the CNs of type $i$, respectively. 
For the sake of clarity, it is useful to isolate the contribution of the repetition component codes in (5) and the contribution of the SPC component codes in (6), so that

$$
\begin{aligned}
I_{E, V}(p, q) & =\sum_{j \geq 2}^{(\text {rep })} \lambda_{j}^{(\mathrm{rep})} \cdot\left(1-q p^{j-1}\right)+\sum_{i}^{(\text {gen })} \lambda_{i} I_{E, V}^{(i)}(p, q) \\
& =\sum_{j \geq 2}^{(\text {rep })} \lambda_{j}^{(\text {rep })}-q \lambda_{\text {rep }}(p)+\sum_{i}^{(\text {gen })} \lambda_{i} I_{E, V}^{(i)}(p, q) \\
I_{E, C}(p) & =\sum_{j \geq 2}^{(\mathrm{SPC})} \rho_{j}^{(\mathrm{SPC})} \cdot(1-p)^{j-1}+\sum_{i}^{(\text {gen })} \rho_{i} I_{E, C}^{(i)}(p) \\
& =\rho_{\mathrm{SPC}}(1-p)+\sum_{i}^{(\text {gen })} \rho_{i} I_{E, C}^{(i)}(p) .
\end{aligned}
$$

In (7), $j$ is the length of the generic repetition $\mathrm{VN}, \lambda_{j}^{(\text {rep })}$ is the fraction of edges connected to the repetition VNs of length $j$, and $\lambda_{\text {rep }}(x) \triangleq \sum_{j \geq 2} \lambda_{j}^{(\text {rep })} x^{j-1}$. We use in (7) the well known EXIT function expression over the BEC for a $(j, 1)$ repetition $\mathrm{VN}$, i.e. $I_{E}(p, q)=1-q p^{j-1}$. The second summation in (7) is over all the generalized VN types. Analogously, in (8) $j$ is the length of the generic SPC CN, $\rho_{j}^{(\mathrm{SPC})}$ is the fraction of edges towards the SPC CNs of length $j, \rho_{\mathrm{SPC}}(x) \triangleq \sum_{j \geq 2} \rho_{j}^{(\mathrm{SPC})} x^{j-1}$, and we use the well-known EXIT function expression over the BEC for a $(j, j-1)$ SPC CN, i.e. $I_{E}(p)=(1-p)^{j-1}$.

The EXIT function of an $(n, k)$ generalized VN over the BEC, when maximum a posteriori (MAP) erasure correction is performed at the $\mathrm{VN}$, can be expressed as

$$
I_{E}(p, q)=1-\frac{1}{n} \sum_{t=0}^{n-1} \sum_{z=0}^{k} a_{t, z} p^{t}(1-p)^{n-t-1} q^{z}(1-q)^{k-z},
$$

which can be readily obtained from [10, eq. 36] with

$$
a_{t, z}=\left[(n-t) \tilde{e}_{n-t, k-z}-(t+1) \tilde{e}_{n-t-1, k-z}\right] .
$$

The parameter $\tilde{e}_{g, h}$ (with $g=0, \ldots, n$ and $h=0, \ldots, k$ ) is known as the $(g, h)$-th un-normalized split information function, defined as explained next. Considering a representation $G$ of the generator matrix for the $(n, k) \mathrm{VN}$, and appending to it the $(k \times k)$ identity matrix $\mathbf{I}_{k}, \tilde{e}_{g, h}$ is equal to the summation of the ranks over all the possible submatrices obtained selecting $g$ columns out of $\mathbf{G}$ and $h$ columns out of $\mathbf{I}_{k}$. We remark that the split information functions for a generalized VN, and therefore its MAP EXIT function (9), depend on the chosen generator 
matrix representation [9]. Then, the performance of the overall D-GLDPC code depends on the code representation used for the variable component codes. For the same reason, two generalized VNs associated with the same code, but with different generator matrices (i.e. different mappings between information words and codewords) must be regarded in (7) as VNs of different types.

The EXIT function of a generalized $(n, k) \mathrm{CN}$ over the BEC, when MAP decoding is performed at the $\mathrm{CN}$, can be obtained by letting $q \rightarrow 1$ in (9). The obtained expression, equivalent to $[10$, eq. 40$]$, is

$$
I_{E}(p)=1-\frac{1}{n} \sum_{t=0}^{n-1} a_{t} p^{t}(1-p)^{n-t-1}
$$

with

$$
a_{t}=(n-t) \tilde{e}_{n-t}-(t+1) \tilde{e}_{n-t-1}
$$

For $g=0, \ldots, n, \tilde{e}_{g}$ is known as the $g$-th un-normalized information function of the $(n, k)$ code, a concept first introduced in [11]. It is defined as the summation of the ranks over all the possible submatrices obtained by selecting $g$ columns out of the generator matrix G. As opposed to the split information functions $\tilde{e}_{g, h}$, the information functions $\tilde{e}_{g}$ are independent of the code representation. Thus, different check component code representations are associated with the same EXIT function for the generalized CN. The performance of a GLDPC or D-GLDPC code is then independent of the specific representation of its generalized check component codes.

Let us suppose that a generic $\mathrm{VN}$ is a $(n, k)$ linear block code $\mathcal{C}$, with generator matrix $\mathbf{G}$. We denote by $\mathcal{C}^{\prime}$ the $(n+k, k)$ linear block code generated by $\left[\mathbf{G} \mid \mathbf{I}_{k}\right]$. The generic codeword of $\mathcal{C}$ is denoted by $\mathbf{c}$, while the generic codeword of $\mathcal{C}^{\prime}$ by $\mathbf{c}^{\prime}$. We have $\mathbf{c}^{\prime}=[\mathbf{c} \mid \mathbf{u}]$, where $\mathbf{c}$ and $\mathbf{u}$ must satisfy $\mathbf{c}=\mathbf{u} \mathbf{G}$ : the code $\mathcal{C}^{\prime}$ then depends on the chosen generator matrix representation for $\mathcal{C}$. It is readily shown that $d_{\min }^{\prime} \geq d_{\min }+1$, where $d_{\min }$ and $d_{\min }^{\prime}$ are the minimum distances of $\mathcal{C}$ and $\mathcal{C}^{\prime}$, respectively.

\section{Reducing a Generator Matrix Rank by Column Elimination}

For a given $(n, k)$ linear block code $\mathcal{C}$ and for a given representation $\mathbf{G}$ of its generator matrix,

we denote by $\mathcal{S}_{t}$ a generic submatrix obtained by selecting $t$ columns out of $\mathbf{G}$, and by $\overline{\mathcal{S}}_{t}$ the submatrix composed of the $n-t$ remaining columns. 
Definition 1: We say that $\overline{\mathcal{S}}_{t}$ covers a non-null codeword $\mathbf{c} \in \mathcal{C}$ when there are no ' 1 ' positions of c corresponding to columns belonging to $\mathcal{S}_{t}$.

Example 1: Let us consider a $(7,3)$ simplex code with generator matrix

$$
\mathbf{G}=\left[\begin{array}{lllllll}
1 & 0 & 0 & 1 & 1 & 0 & 1 \\
0 & 1 & 0 & 1 & 0 & 1 & 1 \\
0 & 0 & 1 & 0 & 1 & 1 & 1
\end{array}\right]
$$

and let us denote by $\mathcal{S}_{2}$ the submatrix composed of the last two columns of $\mathrm{G}$. Then, the only non-null codeword covered by $\overline{\mathcal{S}}_{2}$ is $[0,1,1,1,1,0,0]$.

The following theorem states that in order to reduce the rank of a given generator matrix by column elimination, it is necessary and sufficient that the removed pattern of columns covers at least one non-null codeword.

Theorem 1: Let us consider an $(n, k)$ linear block code $\mathcal{C}$. For any generator matrix representation, we have $\operatorname{rank}\left(\mathcal{S}_{t}\right)<k$ if and only if $\overline{\mathcal{S}}_{t}$ covers at least one codeword.

Proof: [Sufficiency] Suppose that $\overline{\mathcal{S}}_{t}$ covers a codeword $\hat{\mathbf{c}}$, and consider a representation $\hat{\mathbf{G}}$ of the generator matrix where $\hat{\mathbf{c}}$ is one of the rows. It follows that removing from $\hat{\mathbf{G}}$ the $n-t$ columns associated with $\overline{\mathcal{S}}_{t}$ reduces the rank because at least one of the rows becomes an all-zero row, so that $\operatorname{rank}\left(\mathcal{S}_{t}\right)<k$. Since any representation of the generator matrix can be obtained from any other representation by row summations only, and since row summations cannot modify the rank of submatrices composed of generator matrix columns, we have $\operatorname{rank}\left(\mathcal{S}_{t}\right)<k$ also for any representation other than $\hat{\mathbf{G}}$.

[Necessity] Conversely, let us suppose that $\operatorname{rank}\left(\mathcal{S}_{t}\right)<k$ for a given generator matrix representation. Using the same argument as for the sufficiency, we observe that this inequality must be satisfied also for any other representation of the generator matrix. As removing $\overline{\mathcal{S}}_{t}$ from any generator matrix leads to a $(k \times t)$ matrix with reduced rank, it must be possible to obtain (from any generator matrix representation) a generator matrix where one or more rows have only ' 0 ' in those positions corresponding to $\mathcal{S}_{t}$. All these rows are non-null codewords of $\mathcal{C}$ covered by $\overline{\mathcal{S}}_{t}$.

Corollary 1: We have $\operatorname{rank}\left(\mathcal{S}_{t}\right)=k$ for all $\mathcal{S}_{t}$ if and only if $n-t<d_{\text {min }}$. 
Proof: [Sufficiency] Let us suppose that $\operatorname{rank}\left(\mathcal{S}_{t}\right)=k$ for all $\mathcal{S}_{t}$. By applying Theorem 1 it follows that no submatrix $\overline{\mathcal{S}}_{t}$ (composed of $n-t$ columns) can cover any codeword. Then $n-t<d_{\min }$.

[Necessity] Conversely, let us suppose that $n-t<d_{\min }$. Then, no submatrix $\overline{\mathcal{S}}_{t}$ (composed of $n-t$ columns) can cover any codeword. By applying Theorem 1 we conclude that $\operatorname{rank}\left(\mathcal{S}_{t}\right)=k$ for all $\mathcal{S}_{t}$.

Example 2: All the codewords of the $(7,3)$ simplex code of Example 1 have Hamming weight 4. As one of these codewords is $[1,1,1,0,0,0,1]$, Theorem 1 guarantees that if we remove the first three and the last column from $\mathrm{G}$ given in Example 1 (or from any matrix obtained performing row summations on $\mathbf{G})$ we obtain a $(3 \times 3)$ matrix with rank smaller than 3 . On the other hand, by Corollary 1 we know that, even if we remove any set of three or less columns, the rank of $\mathrm{G}$ remains unchanged.

In [9] the concept of independent set was introduced. Given a $(k \times n)$ rank- $r$ binary matrix, an independent set of size $s$ is defined as any set of $s$ columns such that removing these columns from the matrix leads to a $(k \times(n-s))$ matrix with a rank smaller than $r$. By Theorem 1 we now state that a necessary and sufficient condition for a set of $s$ columns to be an independent set of a $(k \times n)$ generator matrix is that the $s$ columns cover at least one codeword. Moreover, by Corollary 1 we recognize that any set of $s<d_{\text {min }}$ columns cannot form an independent set for the generator matrix.

\section{Stability Bound And Derivative Matching for GLDPC Codes}

In GLDPC codes all the variable component codes are repetition codes, which in (7) leads to $\sum_{j \geq 2}^{(\mathrm{rep})} \lambda_{j}^{(\mathrm{rep})}=1$. The EXIT function over the BEC for the VND is then given by $I_{E, V}(p, q)=$ $1-q \lambda_{\text {rep }}(x)$. It follows

$$
\left.\frac{\partial I_{E, V}(p, q)}{\partial p}\right|_{p=0}=-q \lambda_{2}^{(\mathrm{rep})}
$$

From (8), the derivative of $I_{E, C}(p)$ at $p=0$ is

$$
\left.\frac{\mathrm{d} I_{E, C}(p)}{\mathrm{d} p}\right|_{p=0}=-\rho_{\mathrm{SPC}}^{\prime}(1)+\left.\sum_{i}^{\text {(gen) }} \rho_{i} \frac{\mathrm{d} I_{E, C}^{(i)}(p)}{\mathrm{d} p}\right|_{p=0} .
$$


In order to develop (12) it is necessary to explicit the derivative of each generalized CN type EXIT function. This task can be performed by exploiting Corollary 1, as explained next.

Consider an $(n, k)$ generalized $\mathrm{CN}$ with EXIT function $I_{E}(p)$ in the form (10). It is readily shown that

$$
\left.\frac{\mathrm{d} I_{E}(p)}{\mathrm{d} p}\right|_{p=0}=\frac{(n-1) a_{0}-a_{1}}{n} .
$$

We have $a_{0}=0$ if and only if the generalized $\mathrm{CN}$ has minimum distance $d_{\min } \geq 2$. In fact, the generator matrix of the check component code is full rank (rank $=k$ ) by definition, so $\tilde{e}_{n}=k$. Furthermore, from Corollary 1, removing any single column from the generator matrix does not reduce the rank if and only if $d_{\min } \geq 2$, in which case we obtain $\tilde{e}_{n-k}=n k$, so that $a_{0}=n \tilde{e}_{n}-\tilde{e}_{n-1}=n k-n k=0$. As recalled in Section I, the hypothesis $d_{\min } \geq 2$ is always assumed in this paper. Then, we can assume $a_{0}=0$.

If $d_{\min } \geq 2$ for the $\mathrm{CN}$ we obtain

$$
\left.\frac{\mathrm{d} I_{E}(p, q)}{\mathrm{d} p}\right|_{p=0}=-\frac{a_{1}}{n}
$$

where $a_{1}=(n-1) \tilde{e}_{n-1}-2 \tilde{e}_{n-2}=k n(n-1)-2 \tilde{e}_{n-2}$. By applying again Corollary 11, we obtain

$$
a_{1}\left\{\begin{array}{l}
=0 \text { if } d_{\min } \geq 3 \\
>0 \text { if } d_{\min }=2 .
\end{array}\right.
$$

If the $\mathrm{CN}$ exhibits a minimum distance $d_{\min } \geq 3$, then removing any pair of columns from the generator matrix does not affect the rank. In this case $2 \tilde{e}_{n-2}=2 k\left(\begin{array}{l}n \\ 2\end{array}\right)=k n(n-1)$, hence $a_{1}=0$.

According to these results, the only generalized CNs that contribute to the summation in the second term of (12) are those characterized by $d_{\text {min }}=2$. By recalling that all the SPC codes have minimum distance 2 , we conclude that (12) only depends on the check component codes with $d_{\min }=2$. The derivative at $p=0$ of the CND EXIT function can be then expressed as

$$
\begin{aligned}
\left.\frac{\mathrm{d} I_{E, C}(p)}{\mathrm{d} p}\right|_{p=0} & =-\rho_{\mathrm{SPC}}^{\prime}(1)-\sum_{i}^{[2]} \rho_{i} \frac{k_{i} n_{i}\left(n_{i}-1\right)-2 \tilde{e}_{n_{i}-2}}{n_{i}} \\
& =-\rho_{\mathrm{SPC}}^{\prime}(1)-\sum_{i}^{[2]} \rho_{i} \frac{2 \Delta_{n-2}^{(i)}}{n_{i}}
\end{aligned}
$$


where the notation $\sum^{[2]}$ is adopted to indicate the summation over those generalized CN types with minimum distance 2 . In (14), we have denoted by $\Delta_{n-2}^{(i)}$ the expression $k_{i} n_{i}\left(n_{i}-1\right) / 2-\tilde{e}_{n_{i}-2}$, that does not depend on the chosen representation for the $i$-th generalized $\mathrm{CN}$ type.

The next theorem states that $\Delta_{n-2}^{(i)}$ is equal to the multiplicity $A_{2}^{(i)}$ of the weight- 2 codewords for the CNs of type $i$.

Theorem 2: For any linear block check component code with minimum distance $d_{\min }=2$, the parameter $\Delta_{n-2}$ equals the multiplicity $A_{2}$ of the $\mathrm{CN}$ codewords with Hamming weight 2, i.e.

$$
\Delta_{n-2}=A_{2} .
$$

Proof: Let $\mathcal{S}_{n-2}$ be the generic $(k \times(n-2))$ matrix obtained by removing 2 columns from (any representation of) the $\mathrm{CN}$ generator matrix. By Corollary 1 we have that either $\operatorname{rank}\left(\mathcal{S}_{n-2}\right)=$ $k$ or $\operatorname{rank}\left(\mathcal{S}_{n-2}\right)=k-1$ : considering a $\mathrm{CN}$ with $d_{\text {min }}=2$, removing any single column cannot reduce the rank so that removing two columns can reduce the rank at most by one.

We have

$$
\begin{aligned}
\Delta_{n-2} & =\frac{k n(n-1)}{2}-\tilde{e}_{n-2} \\
& =\sum_{\mathcal{S}_{n-2}} k-\sum_{\mathcal{S}_{n-2}} \operatorname{rank}\left(\mathcal{S}_{n-2}\right) \\
& =\sum_{\mathcal{S}_{n-2}}\left(k-\operatorname{rank}\left(\mathcal{S}_{n-2}\right)\right),
\end{aligned}
$$

where we know that each term in the summation is either equal to 0 or to 1 . By Theorem 1 any such term is equal to 1 if and only if $\overline{\mathcal{S}}_{n-2}$ covers a (necessarily weight-2) codeword.

The derivative at $p=0$ of the inverse CND EXIT function $I_{E, C}^{-1}(p)$ is given by $1 / \mathrm{d} I_{E}(p) /\left.\mathrm{d} p\right|_{p=0}$. Combining (11), (14) and Theorem 2, for GLDPC codes, (4) becomes

$$
q^{*} \leq\left[\lambda_{2}^{(\mathrm{rep})}\left(\rho_{\mathrm{SPC}}^{\prime}(1)+\sum_{i}^{[2]} \rho_{i} \frac{2 A_{2}^{(i)}}{n_{i}}\right)\right]^{-1}
$$


We can further simplify (15) by noting that

$$
\begin{aligned}
\rho_{\mathrm{SPC}}^{\prime}(1) & =\sum_{j}^{(\mathrm{SPC})} \rho_{j}(j-1) \\
& =\sum_{j}^{(\mathrm{SPC})} \rho_{j} \frac{2 A_{2}^{(j)}}{n_{j}}
\end{aligned}
$$

as for a $\operatorname{SPC~CN~} n_{j}=j$ and $A_{2}^{(j)}=\left(\begin{array}{l}j \\ 2\end{array}\right)=j(j-1) / 2$. Hence, (15) can be written in the more compact form

$$
\begin{aligned}
q^{*} & \leq\left[\lambda_{2}^{(\mathrm{rep})} \sum_{i}^{[2]} \frac{2 \rho_{i}}{n_{i}} A_{2}^{(i)}\right]^{-1} \\
& =\left[\lambda_{2}^{(\mathrm{rep})} C\right]^{-1}
\end{aligned}
$$

where

$$
C=\sum_{i}^{[2]} \rho_{i} C_{i} \quad \text { with } \quad C_{i}=\frac{2 A_{2}^{(i)}}{n_{i}},
$$

and where now $\sum^{[2]}$ indicates the summation over all the $d_{\min }=2$ check component codes, both SPC and generalized.

For GLDPC codes satisfying the derivative matching condition (3) (the first occurrence of a tangency point between $I_{E, V}(p, q)$ and $I_{E, C}^{-1}(p)$ appears at $\left.p=0\right)$, the threshold assumes the simple closed form $q^{*}=\left[\lambda_{2}^{(\mathrm{rep})} C\right]^{-1}$. If only generalized CNs with $d_{\min } \geq 3$ are used, then (15) becomes $q^{*} \leq\left[\lambda_{2}^{(\text {rep })} \rho_{\text {SPC }}^{\prime}(1)\right]^{-1}$. If the derivative matching condition is fulfilled in this case, we obtain $q^{*}=\left[\lambda_{2}^{(\text {rep })} \rho_{\mathrm{SPC}}^{\prime}(1)\right]^{-1}$.

\section{Stability Bound And Derivative Matching for D-GLDPC Codes}

The derivative at $p=0$ of the CND EXIT function of D-GLDPC codes is the same as for GLDPC codes, that is $\mathrm{d} I_{E}^{-1}(p) /\left.\mathrm{d} p\right|_{p=0}=-1 / C$. The partial derivative of the VND EXIT function with respect to $p$ and evaluated at $p=0$, is developed next.

It follows from (7) that

$$
\left.\frac{\partial I_{E, V}(p, q)}{\partial p}\right|_{p=0}=-q \lambda_{2}^{(\mathrm{rep})}+\left.\sum_{i}^{\text {(gen) }} \lambda_{i} \frac{\partial I_{E, V}^{(i)}(p, q)}{\partial p}\right|_{p=0} .
$$

In order to develop the summation over the generalized VN types in the second part of (17), we have to explicit the partial derivative respect to $p$ of each generalized VN type EXIT function, 
evaluated at $p=0$. To this end, let us consider an $(n, k)$ generalized VN whose EXIT function is given by (9). After defining

$$
f(p)=\sum_{t=0}^{n-1} a_{t, z} p^{t}(1-p)^{n-1-t}
$$

we have

$$
\begin{aligned}
\left.\frac{\partial I_{E}(p, q)}{\partial p}\right|_{p=0} & =-\frac{1}{n} \sum_{z=0}^{k}\left(\left.\frac{\mathrm{d} f(p)}{\mathrm{d} p}\right|_{p=0}\right) q^{z}(1-q)^{k-z} \\
& =\sum_{z=0}^{k} \frac{(n-1) a_{0, z}-a_{1, z}}{n} q^{z}(1-q)^{k-z},
\end{aligned}
$$

as it is readily shown that that $\mathrm{d} f(p) /\left.\mathrm{d} p\right|_{p=0}=-(n-1) a_{0, z}+a_{1, z}$. The expression (18) can be further developed by invoking Corollary 1, Since any variable component code has minimum distance $d_{\min } \geq 2$ by hypothesis, removing any single column from the generator matrix $\mathbf{G}$ of the variable component code cannot reduce the rank of $\mathrm{G}$. It follows

$$
\begin{aligned}
a_{0, z} & =n \tilde{e}_{n, k-z}-\tilde{e}_{n-1, k-z} \\
& =k n\left(\begin{array}{c}
k \\
k-z
\end{array}\right)-k n\left(\begin{array}{c}
k \\
k-z
\end{array}\right) \\
& =0
\end{aligned}
$$

thus leading to

$$
\left.\frac{\partial I_{E}(p, q)}{\partial p}\right|_{p=0}=-\sum_{z=0}^{k} \frac{a_{1, z}}{n} q^{z}(1-q)^{k-z} .
$$

Corollary 1 can be invoked again in order to show that

$$
a_{1, z}\left\{\begin{array}{lll}
=0 & \forall z & \text { if } d_{\min } \geq 3 \\
>0 & \forall z & \text { if } d_{\min }=2,
\end{array}\right.
$$

where $d_{\min }$ is the variable component code minimum distance. In fact, under the hypothesis $d_{\text {min }} \geq 3$, removing any single column or any pair of columns from (any representation of) $\mathbf{G}$ cannot reduce its rank. Under this hypothesis

$$
\begin{aligned}
a_{1, z} & =(n-1) \tilde{e}_{n-1, k-z}-2 \tilde{e}_{n-2, k-z} \\
& =k n(n-1)\left(\begin{array}{c}
k \\
k-z
\end{array}\right)-2 k\left(\begin{array}{c}
n \\
n-2
\end{array}\right)\left(\begin{array}{c}
k \\
k-z
\end{array}\right) \\
& =0 .
\end{aligned}
$$


Hence, the only generalized variable component codes contributing to (17) are those with minimum distance $d_{\min }=2$. This is coherent with the fact that, among the repetition component codes, only those with $d_{\min }=2$ (i.e. the length- 2 repetition codes) give a non-null contribution to (17).

Then, (17) can be developed as

$$
\begin{aligned}
\left.\frac{\partial I_{E, V}(p, q)}{\partial p}\right|_{p=0} & =-q \lambda_{2}^{\text {(rep })}-\sum_{i}^{[2]} \lambda_{i} \sum_{z=0}^{k_{i}} \frac{k_{i} n_{i}\left(n_{i}-1\right)\left(\begin{array}{c}
k_{i} \\
k_{i}-z
\end{array}\right)-2 \tilde{e}_{n_{i}-2, k_{i}-z}}{n_{i}} q^{z}(1-q)^{k_{i}-z} \\
& =-q \lambda_{2}^{(\mathrm{rep})}-\sum_{i}^{[2]} \lambda_{i} \sum_{z=0}^{k_{i}} \frac{2 \Delta_{n-2, k-z}^{(i)}}{n_{i}} q^{z}(1-q)^{k_{i}-z}
\end{aligned}
$$

In the previous expression the symbol $\sum^{[2]}$ indicates the summation over those generalized VN types with minimum distance 2. Moreover, $\Delta_{n-2, k-z}^{(i)}$ is defined as $\frac{k_{i} n_{i}\left(n_{i}-1\right)}{2}\left(\begin{array}{c}k_{i} \\ k_{i}-z\end{array}\right)-\tilde{e}_{n_{i}-z, k_{i}-z}$. As opposed to $\Delta_{n-2}^{(i)}$ in (14), $\Delta_{n-2, k-z}^{(i)}$ in (20) depends on the component code representation.

Using (20) we can express (4) for a D-GLDPC code as

$$
q^{*} \lambda_{2}^{(\mathrm{rep})}+\sum_{i}^{[2]} \lambda_{i} \sum_{z=0}^{k_{i}} \frac{2 \Delta_{n-2, k-z}^{(i)}}{n_{i}}\left(q^{*}\right)^{z}\left(1-q^{*}\right)^{k_{i}-z} \leq \frac{1}{C} .
$$

In the reminder of this section, we prove that (21) can be written as an explicit upper bound to the decoding threshold $q^{*}$. We start by proving the following theorem.

Theorem 3: Let us consider an $(n, k)$ linear block variable component code with minimum distance $d_{\min }=2$. We have

$$
\sum_{z=0}^{k} \frac{2 \Delta_{n-2, k-z}}{n}\left(q^{*}\right)^{z}\left(1-q^{*}\right)^{k-z}=\sum_{u=1}^{k} \frac{2 A_{2, u}}{n}\left(q^{*}\right)^{u},
$$

where $A_{2, u}$ is the number of the $\mathrm{VN}$ weight- 2 codewords generated by weight- $u$ information words.

Proof: Let $\mathcal{C}$ be the $(n, k)$ variable component code and let $\mathbf{G}$ be the chosen generator matrix for $\mathcal{C}$. Moreover, let $\mathcal{S}_{n-2, k-z}$ be the generic $(k \times(n-2+k-z))$ matrix obtained by selecting $n-2$ columns in $\mathbf{G}$ and $k-z$ columns in the $(k \times k)$ identity matrix.

Let us apply Theorem 1 to the code $\mathcal{C}^{\prime}$ introduced at the end of Section II Each codeword $\mathbf{c}^{\prime} \in \mathcal{C}^{\prime}$ is composed of the concatenation of a codeword $\mathbf{c} \in \mathcal{C}$ with one of the possible $2^{k}$ sequences of $k$ bits (where by the linearity of $\mathcal{C}$ the all-zero length- $k$ sequence is always concatenated with the all-zero codeword of $\mathcal{C}$ ). Combining this observation with Theorem 1 
and introducing the notation $\mathcal{S}_{n-2, k-z}=\left[\mathcal{S}_{n-2}^{\mathrm{G}} \mid \mathcal{S}_{k-z}^{\mathrm{I}}\right]$ we observe that a necessary (though not sufficient) condition for having $\operatorname{rank}\left(\mathcal{S}_{n-2, k-z}\right)<k$ is that $\overline{\mathcal{S}}_{n-2}^{\mathrm{G}}$ covers a weight-2 codeword of $\mathcal{C}$.

Next, we develop $\Delta_{n-2, k-z}$ as

$$
\begin{aligned}
\Delta_{n-2, k-z} & =\frac{k n(n-1)}{2}\left(\begin{array}{c}
k \\
k-z
\end{array}\right)-\tilde{e}_{n-2, k-z} \\
& =\sum_{\mathcal{S}_{n-2, k-z}} k-\sum_{\mathcal{S}_{n-2, k-z}} \operatorname{rank}\left(\mathcal{S}_{n-2, k-z}\right) \\
& =\sum_{\mathcal{S}_{n-2, k-z}}\left(k-\operatorname{rank}\left(\mathcal{S}_{n-2, k-z}\right)\right) \\
& =\sum_{\mathcal{S}_{n-2}^{\mathbf{G}}} \sum_{\mathcal{S}_{k-z}^{\mathbf{I}}}\left(k-\operatorname{rank}\left(\left[\mathcal{S}_{n-2}^{\mathbf{G}} \mid \mathcal{S}_{k-z}^{\mathbf{I}}\right]\right)\right) \\
& =\sum_{\mathbf{c}}^{[2]} \sum_{\mathcal{S}_{k-z}^{\mathbf{I}}}\left(k-\operatorname{rank}\left(\left[\mathcal{S}_{n-2}^{\mathbf{G}} \mid \mathcal{S}_{k-z}^{\mathbf{I}}\right]\right)\right)
\end{aligned}
$$

where $\sum_{\mathbf{c}}^{[2]}$ is used to indicate the summation over those $\mathcal{S}_{n-2}^{\mathrm{G}}$ such that $\overline{\mathcal{S}}_{n-2}^{\mathrm{G}}$ covers a weight-2 codeword of $\mathcal{C}$. Then, we can write

$$
\begin{aligned}
\sum_{z=0}^{k} \frac{2 \Delta_{n-2, k-z}}{n} & \left(q^{*}\right)^{z}\left(1-q^{*}\right)^{k-z} \\
& =\frac{2}{n} \sum_{z=0}^{k} \sum_{\mathbf{c}}^{[2]} \sum_{\mathcal{S}_{k-z}^{\mathbf{I}}}\left(k-\operatorname{rank}\left(\left[\mathcal{S}_{n-2}^{\mathbf{G}} \mid \mathcal{S}_{k-z}^{\mathbf{I}}\right]\right)\right)\left(q^{*}\right)^{z}\left(1-q^{*}\right)^{k-z} \\
& =\frac{2}{n} \sum_{\mathbf{c}}^{[2]} \sum_{z=0}^{k} \sum_{\mathcal{S}_{k-z}^{\mathbf{I}}}\left(k-\operatorname{rank}\left(\left[\mathcal{S}_{n-2}^{\mathbf{G}} \mid \mathcal{S}_{k-z}^{\mathbf{I}}\right]\right)\right)\left(q^{*}\right)^{z}\left(1-q^{*}\right)^{k-z}
\end{aligned}
$$

By hypothesis there are no VNs with minimum distance 1. Then, for a given weight-2 codeword c $\in \mathcal{C}$, any submatrix $\mathcal{S}_{n-2, k-z}$ is such that $\overline{\mathcal{S}}_{n-2, k-z}$ can cover at most one codeword of $\mathcal{C}^{\prime}$, i.e. the codeword $\left[\mathbf{c} \mid \mathbf{u}_{\mathbf{c}}\right]$ subject to $\mathbf{c}=\mathbf{u}_{\mathbf{c}} \mathbf{G}$. If we denote by $w_{H}\left(\mathbf{u}_{\mathbf{c}}\right)$ the Hamming weight of $\mathbf{u}_{\mathbf{c}}$, for each weight-2 codeword $\mathbf{c} \in \mathcal{C}$ the summation over $z$ in (23) can always start from $w_{H}\left(\mathbf{u}_{\mathbf{c}}\right)$. In fact, for $z=0, \ldots, w_{H}\left(\mathbf{u}_{\mathbf{c}}\right)-1$ it is not possible for $\overline{\mathcal{S}}_{n-2, k-z}$ to cover the codeword $\left[\mathbf{c} \mid \mathbf{u}_{\mathbf{c}}\right]$, so that $k-\operatorname{rank}\left(\left[\mathcal{S}_{n-2}^{\mathbf{G}} \mid \mathcal{S}_{k-z}^{\mathbf{I}}\right]\right)=0$. That allows writing the second member 
in (23) as

$$
\frac{2}{n} \sum_{\mathbf{c}}^{[2]} \sum_{z=w_{H}\left(\mathbf{u}_{\mathbf{c}}\right)}^{k} \sum_{\mathcal{S}_{k-z}^{\mathbf{I}}}\left(k-\operatorname{rank}\left(\left[\mathcal{S}_{n-2}^{\mathbf{G}} \mid \mathcal{S}_{k-z}^{\mathbf{I}}\right]\right)\right)\left(q^{*}\right)^{z}\left(1-q^{*}\right)^{k-z} .
$$

For given $z \geq w_{H}\left(\mathbf{u}_{\mathbf{c}}\right)$, the codeword $\left[\mathbf{c} \mid \mathbf{u}_{\mathbf{c}}\right]$ is covered by exactly $\left(\begin{array}{l}k-w_{H}\left(\mathbf{u}_{\mathbf{c}}\right) \\ z-w_{H}\left(\mathbf{u}_{\mathbf{c}}\right)\end{array}\right)$ matrices $\overline{\mathcal{S}}_{n-2, k-z}$. Hence, there are exactly $\left(\begin{array}{l}k-w_{H}\left(\mathbf{u}_{\mathbf{c}}\right) \\ z-w_{H}\left(\mathbf{u}_{\mathbf{c}}\right)\end{array}\right)$ non-null terms in

$$
\sum_{\mathcal{S}_{k-z}^{\mathrm{I}}}\left(k-\operatorname{rank}\left(\mathcal{S}_{n-2}^{\mathbf{G}} \mid \mathcal{S}_{k-z}^{\mathbf{I}}\right)\right)\left(q^{*}\right)^{z}\left(1-q^{*}\right)^{k-z} .
$$

Deleting from $\mathrm{G}$ two columns corresponding to a weight-2 codeword of $\mathcal{C}$ reduces the rank of this matrix by one, leading to a rank $k-1$. In fact, considering the $\mathrm{VN}$ minimum distance $d_{\text {min }}=2$, removing the first column cannot reduce the rank (Corollary 1) and removing the second column reduces the rank (Theorem 1) necessarily by one. We can then conclude that each of the $\left(\begin{array}{c}k-w_{H}\left(\mathbf{u}_{\mathbf{c}}\right) \\ z-w_{H}\left(\mathbf{u}_{\mathbf{c}}\right)\end{array}\right)$ non-null terms in the summation $\sum_{\mathcal{S}_{k-z}^{\mathbf{I}}}\left(k-\operatorname{rank}\left(\left[\mathcal{S}_{n-2}^{\mathbf{G}} \mid \mathcal{S}_{k-z}^{\mathbf{I}}\right]\right)\right)$ is equal to one, independently of $z$. Then we can further develop (24) as

$$
\frac{2}{n} \sum_{\mathbf{c}}^{[2]} \sum_{z=w_{H}\left(\mathbf{u}_{\mathbf{c}}\right)}^{k}\left(\begin{array}{l}
k-w_{H}\left(\mathbf{u}_{\mathbf{c}}\right) \\
z-w_{H}\left(\mathbf{u}_{\mathbf{c}}\right)
\end{array}\right)\left(q^{*}\right)^{z}\left(1-q^{*}\right)^{k-z} .
$$

We next observe that those weight- 2 codewords $\mathbf{c} \in \mathcal{C}$ associated with the same $w_{H}\left(\mathbf{u}_{\mathbf{c}}\right)$ (i.e. generated by information words having the same weight) produce the same contribution in (25), since only the Hamming weight of the information words $\mathbf{u}_{\mathbf{c}}$ matters. This observation allows us to write (25) as

$$
\sum_{u=1}^{k} \frac{2 A_{2, u}}{n} \sum_{z=u}^{k}\left(\begin{array}{l}
k-u \\
z-u
\end{array}\right)\left(q^{*}\right)^{z}\left(1-q^{*}\right)^{k-z},
$$

where $A_{2, u}$ is the number of weight-2 codewords $\mathbf{c} \in \mathcal{C}$ such that $w_{H}\left(\mathbf{u}_{\mathbf{c}}\right)=u$. In general, $A_{2, u}$ depends on the variable component code representation. By noting that

$$
\sum_{z=u}^{k}\left(\begin{array}{c}
k-u \\
z-u
\end{array}\right)\left(q^{*}\right)^{z}\left(1-q^{*}\right)^{k-z}=\left(q^{*}\right)^{u}
$$

we finally obtain (22).

Theorem 3 allows us to write the first member of (21) as

$$
q^{*} \lambda_{2}^{(\text {rep })}+\sum_{i}^{[2]} \lambda_{i} \sum_{u=1}^{k_{i}} \frac{2 A_{2, u}^{(i)}}{n_{i}}\left(q^{*}\right)^{u}
$$


The length-2 repetition VNs can be embedded into the summation over the generalized VN types with minimum distance 2 . In fact, the only weight-2 codeword of a length-2 repetition $\mathrm{VN}$ is $\mathbf{c}=[1,1]$, which is generated by a weight-1 information word. Then, for a length-2 repetition VN we have

$$
\lambda_{2}^{\text {(rep) }} \sum_{u=1}^{k} \frac{2 A_{2, u}}{n}\left(q^{*}\right)^{u}=\lambda_{2}^{\text {(rep) }} q^{*} .
$$

Hence, (21) can be put into the more compact form

$$
\sum_{i}^{[2]} \lambda_{i} \sum_{u=1}^{k_{i}} \frac{2 A_{2, u}^{(i)}}{n_{i}}\left(q^{*}\right)^{u} \leq \frac{1}{C}
$$

where now the summation $\sum^{[2]}$ is over all the VN types with minimum distance 2, both repetition and generalized.

The first part of (27) is a real polynomial $P(\cdot)$ in the variable $q^{*}$. This polynomial can be written as $P(x)=\sum_{i}^{[2]} \lambda_{i} P_{i}(x)$, where $P_{i}(\cdot)$ is a degree- $k_{i}$ real polynomial associated with the $d_{\text {min }}=2$ type- $i$ VNs. Each $P_{i}(\cdot)$ is a monotonically increasing function (since all its coefficients are positive). Consequently, $P(\cdot)$ is a monotonically increasing function and its inverse $P^{-1}(\cdot)$ exists. We have then proved the following theorem, which is the main contribution of this paper.

Theorem 4 (Stability bound over the BEC for D-GLDPC codes): The asymptotic threshold $q^{*}$ of a D-GLDPC code ensemble over the BEC, assuming MAP erasure correction at each component code, fulfills

$$
q^{*} \leq P^{-1}\left(\frac{1}{C}\right)
$$

where

$$
P(x)=\sum_{i}^{[2]} \lambda_{i} P_{i}(x) \quad \text { with } \quad P_{i}(x)=\sum_{u=1}^{k_{i}} \frac{2 A_{2, u}^{(i)}}{n_{i}} x^{u}
$$

and

$$
C=\sum_{i}^{[2]} \rho_{i} C_{i} \quad \text { with } \quad C_{i}=\frac{2 A_{2}^{(i)}}{n_{i}}
$$

For an LDPC code ensemble (28) returns $q^{*} \leq\left[\lambda^{\prime}(0) \rho^{\prime}(1)\right]^{-1}$, i.e. the well-known stability bound for LDPC codes. 
Property 1: For a length-2 repetition VN (i.e. for a conventional LDPC degree-2 VN) we have $P_{i}(x)=x$. Hence, if the only $d_{\min }=2 \mathrm{VNs}$ are length-2 repetition codes, we have $P^{-1}(x)=\left(1 / \lambda_{2}^{(\mathrm{rep})}\right) x$. This is the case for GLDPC codes.

Property 2: For a length- $n_{i}$ SPC CN (i.e. for a conventional LDPC degree- $n_{i} \mathrm{CN}$ ) we have $C_{i}=n_{i}-1$.

Property 3: Any length- $n_{i}$ and weight- 2 binary sequence is a codeword for a length- $n_{i}$ SPC $\mathrm{CN}$. Then, $C_{i}$ for a length- $n_{i} \mathrm{CN}$ with minimum distance 2 is maximum when the $\mathrm{CN}$ is a SPC code. In other words, $C_{i}$ fulfills

$$
C_{i} \leq n_{i}-1
$$

where the equality holds when the $\mathrm{CN}$ is a SPC code.

Property 4: For any VN with minimum distance $d_{\min }=2$, the value of $P_{i}(x)$ depends on the chosen generator matrix through the coefficients $A_{2, u}^{(i)}$. This is true for all $x$, except at $x=0$ and at $x=1$ where we have

$$
P_{i}(0)=0
$$

and

$$
P_{i}(1)=\frac{2 A_{2}^{(i)}}{n_{i}}=C_{i}
$$

respectively. Independently of the $\mathrm{VN}$ representation, the value assumed by $P_{i}(x)$ at $x=1$ is equal to the value of $C_{i}$ for the same $d_{\min }=2$ linear block code when used as a $\mathrm{CN}$.

Property 5: For a D-GLDPC code ensemble satisfying the derivative matching condition, the iterative decoding threshold over the BEC assumes the simple form $q^{*}=P^{-1}(1 / C)$.

It should be noted that in general $P^{-1}(1 / C)$ is not a closed form for the threshold. However, there are simple cases in which $P^{-1}(\cdot)$ can be explicited. An example is provided in the appendix.

\section{CONCLUSION}

In this paper, a stability bound over the BEC has been developed for D-GLDPC codes. It generalizes the inequality $q^{*} \leq\left[\lambda^{\prime}(0) \rho^{\prime}(1)\right]^{-1}$, valid for LDPC code ensembles. We have shown 
that for D-GLDPC codes, as for LDPC codes, the only variable and check component codes contributing to the bound are those having minimum distance 2. A derivative matching condition sufficient to achieve the bound with equality has also been defined. If the derivative matching condition is fulfilled, then the decoding threshold over the BEC for D-GLDPC codes is expressed by a simple formula, although in general not in closed-form. For GLDPC codes this formula always leads to a closed-form threshold expression.

\section{APPENDIX I}

\section{D-GLDPC CODES WITH SPC VARIABLE NODES}

GLDPC codes employing strong generalized CNs (such as Hamming or BCH CNs) represent a possible solution for obtaining a good compromise between waterfall performance and error floor. Examples of such GLDPC code constructions are described in [12]-[16]. In general, increasing the fraction of strong generalized $\mathrm{CNs}$ can be very favorable from the point of view of the overall code minimum distance and then of the error floor, but presents drawbacks.

A first drawback is represented by an overall code rate loss which makes GLDPC codes with large fractions of strong generalized CNs of interest only for low or very low rate [17]. The reason is briefly reviewed next. Let us consider a more general code structure, namely a D-GLDPC code. If we denote by $r_{V, i}$ and by $r_{C, j}$ the code rate of the type- $i$ VNs and of the type- $j \mathrm{CNs}$, respectively, the overall design rate is

$$
R=1-\frac{\sum_{j} \rho_{j}\left(1-r_{C, j}\right)}{\sum_{i} \lambda_{i} r_{V, i}},
$$

which is monotonically increasing respect to any $r_{V, i}$ and to any $r_{C, j}$. A generalized $\mathrm{CN}$ of length $n$ has a code rate smaller than the code rate of a length- $n$ SPC CN. Then, a large fraction of strong generalized CNs determines an overall rate loss. In GLDPC codes this rate loss is difficult to compensate even using large fractions of length-2 repetition VNs (which are the highest rate VNs available if all the node in the Tanner graph have minimum distance at least 2) so that usually the overall GLDPC code remains of low rate. A second drawback is that GLDPC codes with large fractions of strong generalized CNs and large fractions of length-2 repetition VN are typically characterized by a poor asymptotic threshold due to the large area gap between the EXIT curves in the EXIT function (see the Area Theorem in [18]).

Allowing the generalization of the VND together with the generalization of the CND provides an increased flexibility in the code design, that can be exploited to overcome the above mentioned 
limitations. In particular, the rate loss due to the generalized CNs can be compensated using generalized VNs with a code rate larger than 1/2. In this context, a special class of generalized VNs is represented by $(n, n-1)$ SPC VNs each one having $n$ edges towards the CND and associated with $n-1$ encoded bits. It is shown in [19] that these codes can be effectively exploited for the design of D-GLDPC codes with good waterfall and error floor performance. In this appendix, we develop the polynomial $P_{i}(\cdot)$ defined in Theorem 4 for such VNs when represented in both systematic and cyclic form. We also propose a numerical example illustrating the capabilities offered by D-GLDPC codes with SPC VNs.

\section{A. SPC Variable Nodes in Systematic Form}

Let us suppose that the VNs of type- $i$ are length- $n_{i}$ SPC codes in systematic form, i.e., represented by the $\left(\left(n_{i}-1\right) \times n_{i}\right)$ generator matrix

$$
\mathbf{G}_{i}=\left[\begin{array}{cccccc}
1 & 0 & 0 & \ldots & 0 & 1 \\
0 & 1 & 0 & \ldots & 0 & 1 \\
0 & 0 & 1 & \ldots & 0 & 1 \\
\vdots & \vdots & \vdots & \ddots & \vdots & \vdots \\
0 & 0 & 0 & \ldots & 1 & 1
\end{array}\right]
$$

Each of these VNs has $\left(\begin{array}{c}n_{i} \\ 2\end{array}\right)$ weight-2 codewords. Specifically, there are $n_{i}-1$ weight- 2 codewords generated by weight- 1 information words, $\left(\begin{array}{c}n_{i}-1 \\ 2\end{array}\right)=\frac{\left(n_{i}-1\right)\left(n_{i}-2\right)}{2}$ weight- 2 codewords generated by weight- 2 information words and no weight- 2 codewords generated by information words of weight larger than 2. Then

$$
A_{2, u}^{(i)}=\left\{\begin{array}{lll}
n_{i}-1 & \text { if } u=1 \\
\left(n_{i}-1\right)\left(n_{i}-2\right) / 2 & \text { if } u=2 \\
0 & \text { if } u=3, \ldots, n_{i}-1
\end{array}\right.
$$

so that

$$
\begin{aligned}
P_{i}(x) & =\frac{2}{n_{i}} \cdot\left(n_{i}-1\right) x+\frac{2}{n_{i}} \cdot \frac{\left(n_{i}-1\right)\left(n_{i}-2\right)}{2} x^{2} \\
& =\frac{2\left(n_{i}-1\right)}{n_{i}} x\left(1+\frac{n_{i}-2}{2} x\right) .
\end{aligned}
$$


B. SPC Variable Nodes in Cyclic Form

Let the VNs of type- $i$ be $\left(n_{i}, n_{i}-1\right)$ SPC codes in cyclic form, i.e. generated by

$$
\mathbf{G}_{i}=\left[\begin{array}{cccccc}
1 & 1 & 0 & \ldots & 0 & 0 \\
0 & 1 & 1 & \ldots & 0 & 0 \\
0 & 0 & 1 & \ldots & 0 & 0 \\
\vdots & \vdots & \vdots & \ddots & \vdots & \vdots \\
0 & 0 & 0 & \ldots & 1 & 1
\end{array}\right]
$$

In this case we obtain an expression of $P_{i}(x)$ different from (30). In fact, it is readily shown that in a SPC code represented in cyclic form, an information word of weight $u$ generates a weight- 2 codeword if and only if all its ' 1 ' positions are contiguous. Then, for all $u=1, \ldots, n_{i}-1$ we have $A_{2, u}^{(i)}=n_{i}-u$, from which

$$
\begin{aligned}
P_{i}(x) & =\sum_{u=1}^{n_{i}-1} \frac{2\left(n_{i}-u\right)}{n_{i}} x^{u} \\
& =2 \sum_{u=1}^{n_{i}-1} x^{u}-\frac{2}{n_{i}} \sum_{u=1}^{n_{i}-1} u x^{u} \\
& =2 x \frac{x^{n_{i}-1}-1}{x-1}-\frac{2 x}{n_{i}} \cdot \frac{1-n_{i} x^{n_{i}-1}+\left(n_{i}-1\right) x^{n_{i}}}{(x-1)^{2}} \\
& =\frac{2 x\left[x^{n_{i}}-n_{i}(x-1)-1\right]}{n_{i}(x-1)^{2}} .
\end{aligned}
$$

If $n_{i}=2$ or $n_{i}=3$, then (30) coincides with (31) as expected. Specifically, from both (30) and (31) we obtain $P_{i}(x)=x$ and $P_{i}(x)=\frac{2}{3} x^{2}+\frac{4}{3} x$ for $n_{i}=2$ and $n_{i}=3$, respectively.

\section{Comparison between Systematic and Cyclic Form}

Let us denote by $P_{s}(\cdot)$ and by $P_{c}(\cdot)$ the polynomial $P_{i}(\cdot)$ of a length- $n$ SPC VN in systematic and cyclic form, respectively. We show next that if $n>3$

$$
P_{s}(x)-P_{c}(x)\left\{\begin{array}{lll}
>0 & \text { if } & 0<x<1 \\
=0 & \text { if } & x=1 \\
<0 & \text { if } & x>1
\end{array}\right.
$$


In fact, we have

$$
\begin{aligned}
P_{s}(x)-P_{c}(x) & =\frac{2}{n}\left[(n-1) x+\frac{(n-1)(n-2)}{2} x^{2}\right]-\frac{2}{n} \sum_{u=1}^{n-1}(n-u) x^{u} \\
& =\frac{2 x^{2}}{n}\left[\frac{(n-2)(n-3)}{2}-\sum_{u=3}^{n-1}(n-u) x^{u-2}\right] .
\end{aligned}
$$

It is readily shown that $\sum_{u=3}^{n-1}(n-u)=\frac{(n-2)(n-3)}{2}$. Then, $P_{s}(1)-P_{c}(1)=0$, a result which is consistent with Property 4. For $0<x<1$ we must have $\sum_{u=3}^{n-1}(n-u) x^{u-2}<\frac{(n-2)(n-3)}{2}$ which leads to $P_{s}(x)-P_{c}(x)>0$; analogously, for $x>1$ we must have $\sum_{u=3}^{n-1}(n-u) x^{u-2}>\frac{(n-2)(n-3)}{2}$ which leads to $P_{s}(x)-P_{c}(x)<0$.

\section{D-GLDPC Codes with Length-2 Repetition VNs and SPC VNs in Systematic Form}

Let us consider (28). Although in general it is not possible to express $P^{-1}(\cdot)$ in an explicit closed form, this is possible in special cases. For instance, obtaining a closed form expression of $P^{-1}(\cdot)$ is possible when the only $d_{\min }=2$ variable component codes are length- 2 repetition codes and length- $n$ SPC codes in systematic form. Let $\lambda$ be the fraction of edges connected to the length-2 repetition VNs and $\mu$ the fraction of edges connected to the length- $n$ SPC VNs (so $\lambda+\mu$ is the total fraction of edges connected to $\left.d_{\min }=2 \mathrm{VNs}\right)$. We have

$$
P(x)=\lambda x+\mu \frac{2(n-1)}{n} x\left(\frac{n-2}{2} x+1\right) .
$$

By solving for positive $y$ the equation $P(y)=x$, we obtain

$$
P^{-1}(x)=\frac{-[n \lambda+2(n-1) \mu]}{2(n-2)(n-1) \mu}+\frac{\sqrt{[n \lambda+2(n-1) \mu]^{2}+4(n-2)(n-1) n \mu x}}{2(n-2)(n-1) \mu} .
$$

In Fig. 2, (33) is plotted for different values of $\mu$, assuming $\lambda+\mu=0.3$ and SPC VNs of length $n=7$. Each curve is associated with a different value of $\mu$, i.e., with a different proportion between length-2 repetition VNs and length-7 SPC VNs in the VND. Hence, the curve labelled as 0.0 corresponds to the presence of only length- 2 repetition VNs, while the curve labelled as 0.3 to the presence of only SPC VNs. Hence modifying $\mu$ provides a wide variety of options. 


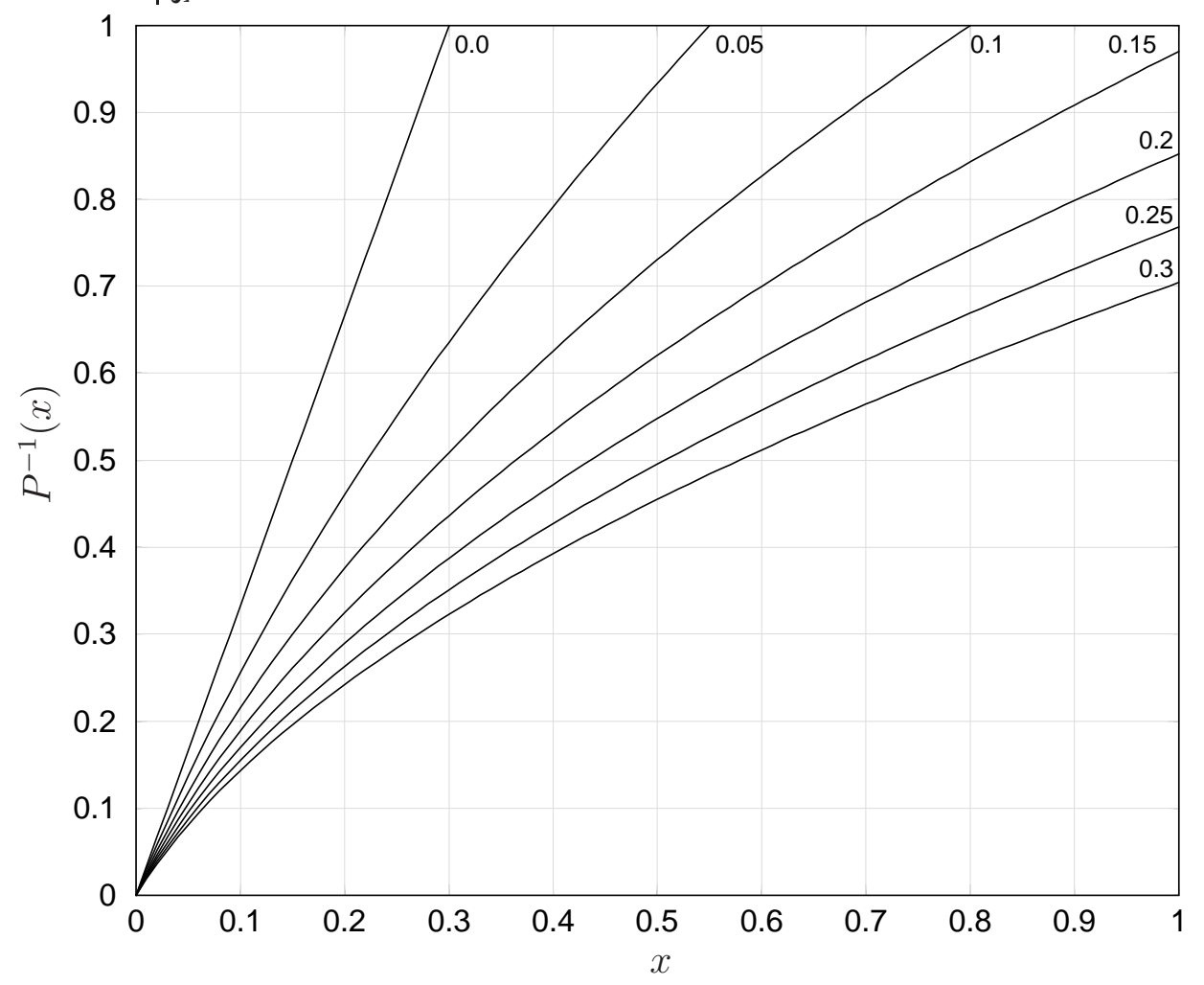

Fig. 2. Plot of $P^{-1}(\cdot)$ for a D-GLDPC code where the only $d_{\min }=2$ VNs are length-2 repetition and length-7 SPC VNs. The total fraction of edges connected to $d_{\min }=2 \mathrm{VNs}$ is $\lambda+\mu=0.3$, and each curve is associated with a specific value of $\mu$.

\section{E. Distribution Optimization}

We consider the optimization problem of a GLDPC and of a D-GLDPC code ensemble for design rate $R=1 / 2$. In both cases we constrain the optimization process by allowing the repetition VN degree to range only between 2 and 15 and the SPC CN degree only between 5 and 15. Moreover, we use $(31,21) \mathrm{BCH}$ CNs, imposing a minimum fraction of edges connected to the $\mathrm{BCH}$ CNs equal to 0.7. For the D-GLDPC code ensemble, we allow also length-15 SPC CNs in cyclic form. The output of an optimization process over the BEC performed with differential evolution [20], [21] is reported in Table I (from an edge perspective). For each of the two optimized distributions the threshold and the stability bound (28) are shown. While for the GLDPC code ensemble it is necessary to use only length-2 repetition VNs to compensate the rate loss introduced by the large fraction of $\mathrm{BCH} \mathrm{CNs}$ with an overall poor threshold, for the D-GLDPC code ensemble the use of SPC VNs allows obtaining a much larger threshold. 
TABLE I

GLDPC AND D-GLDPC DISTRIBUTIONS WITH LARGE FRACTIONS OF BCH CHECK NODES

\begin{tabular}{|l|l|l|}
\hline & GLDPC & D-GLDPC \\
\hline \hline \multicolumn{3}{|c|}{ Variable Nodes } \\
\hline \hline SPC $_{\text {cyc } 15}$ & & 0.521581 \\
rep 2 & 1.000000 & 0.132836 \\
rep 14 & & 0.145293 \\
rep 15 & & 0.200291 \\
\hline \hline \multicolumn{3}{|c|}{ Check Nodes } \\
\hline \hline BCH & 0.700000 & 0.721799 \\
SPC 5 & & 0.278201 \\
SPC 12 & 0.174190 & \\
SPC 13 & 0.125810 & \\
\hline \hline$q^{*}$ & 0.291516 & 0.478585 \\
\hline$P^{-1}(1 / C)$ & 0.291902 & 0.478585 \\
\hline
\end{tabular}

From an EXIT chart perspective the capability of the SPC VNs to reduce the area gap between the EXIT curves is illustrated by comparing in Fig. 3 and Fig. 4 .

\section{REFERENCES}

[1] R. Gallager, Low-Density Parity-Check Codes. Cambridge, Massachussets: M.I.T. Press, 1963.

[2] R. M. Tanner, "A recursive approach to low complexity codes," IEEE Trans. Inform. Theory, vol. 27, no. 5, pp. 533-547, Sept. 1981.

[3] Y. Wang and M. Fossorier, "Doubly generalized low-density parity-check codes," in Proc. of 2006 IEEE International Symposium on Information Theory, Seattle, USA, July 2006, pp. 669-673.

[4] E. Paolini, "Iterative decoding methods based on low-density graphs," Ph.D. dissertation, University of Bologna, Bologna, Italy, May 2007.

[5] M. Luby, M. Mitzenmacher, M. Shokrollahi, and D. Spielman, "Efficient erasure correcting codes," IEEE Trans. Inform. Theory, vol. 47, no. 2, pp. 569-584, Feb. 2001.

[6] T. Richardson, M. Shokrollahi, and R. Urbanke, "Design of capacity-approaching irregular low-density parity-check codes," IEEE Trans. Inform. Theory, vol. 47, no. 2, pp. 619-637, Feb. 2001.

[7] T. Richardson and R. Urbanke, Modern Coding Theory, 2007, (preprint). [Online]. Available: http://lthcwww.epfl.ch/mct/index.php

[8] S. ten Brink, "Convergence behavior of iteratively decoded parallel concatenated codes," IEEE Trans. Commun., vol. 49, no. 10 , pp. 1727-1737, Oct. 2001. 


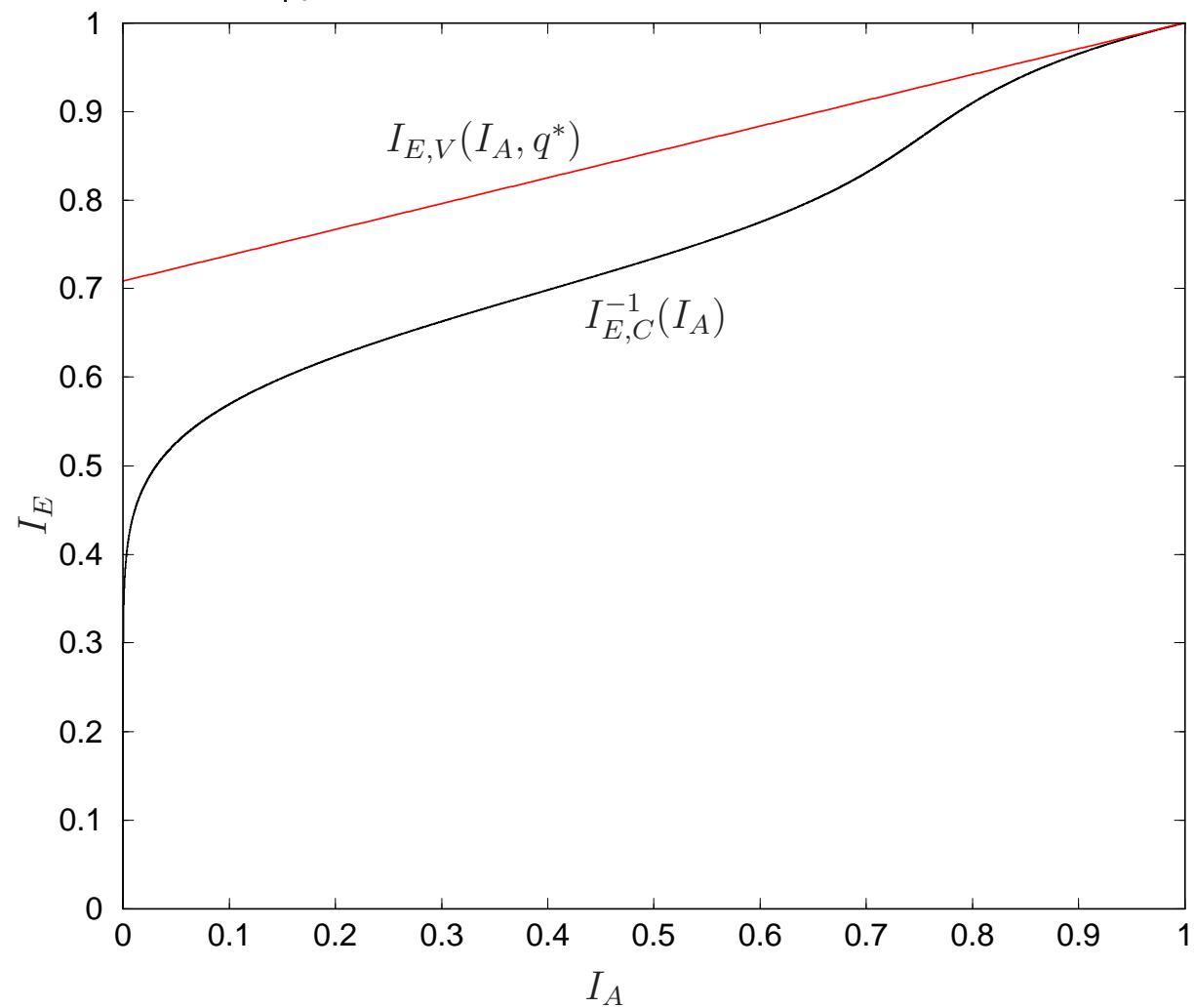

Fig. 3. EXIT chart for the GLDPC distribution in Table I.

[9] E. Paolini, M. Fossorier, and M. Chiani, "Generalized and doubly-generalized LDPC codes with random component codes for the binary erasure channel," IEEE Trans. Inform. Theory, 2007, submitted.

[10] A. Ashikhmin, G. Kramer, and S. ten Brink, "Extrinsic information transfer functions: Model and erasure channel properties," IEEE Trans. Inform. Theory, vol. 50, pp. 2657-2673, Nov. 2004.

[11] T. Helleseth, T. Kløve, and V. I. Levenshtein, "On the information function of an error-correcting code," IEEE Trans. Inform. Theory, vol. 43, pp. 549-557, Mar. 1997.

[12] J. Boutros, O. Pothier, and G. Zemor, "Generalized low density (Tanner) codes," in Proc. of 1999 IEEE International Conference on Communications, vol. 1, Vancouver, Canada, June 1999, pp. 441-445.

[13] M. Lentmaier and K. Zigangirov, "On generalized low-density parity-check codes based on Hamming component codes," IEEE Commun. Lett., vol. 3, no. 8, pp. 248-250, Aug. 1999.

[14] G. Yue, L. Ping, and X. Wang, "Low-rate generalized low-density parity-check codes with Hadamard constraints," in Proc. of 2005 IEEE International Symposium on Information Theory, Adelaide, Australia, Sept. 2005, pp. 1377-1381.

[15] J. Chen and R. Tanner, "A hybrid coding scheme for the Gilbert-Elliott channel," IEEE Trans. Commun., vol. 54, no. 10, pp. 564-571, Oct. 2006.

[16] G. Liva, W. Ryan, and M. Chiani, "Quasi-cyclic generalized LDPC codes with low error floors," IEEE Trans. Commun., vol. 56, no. 1, pp. 49-57, Jan. 2008.

[17] N. Miladinovic and M. Fossorier, "Generalized LDPC codes with Reed-Solomon and BCH codes as component codes for 


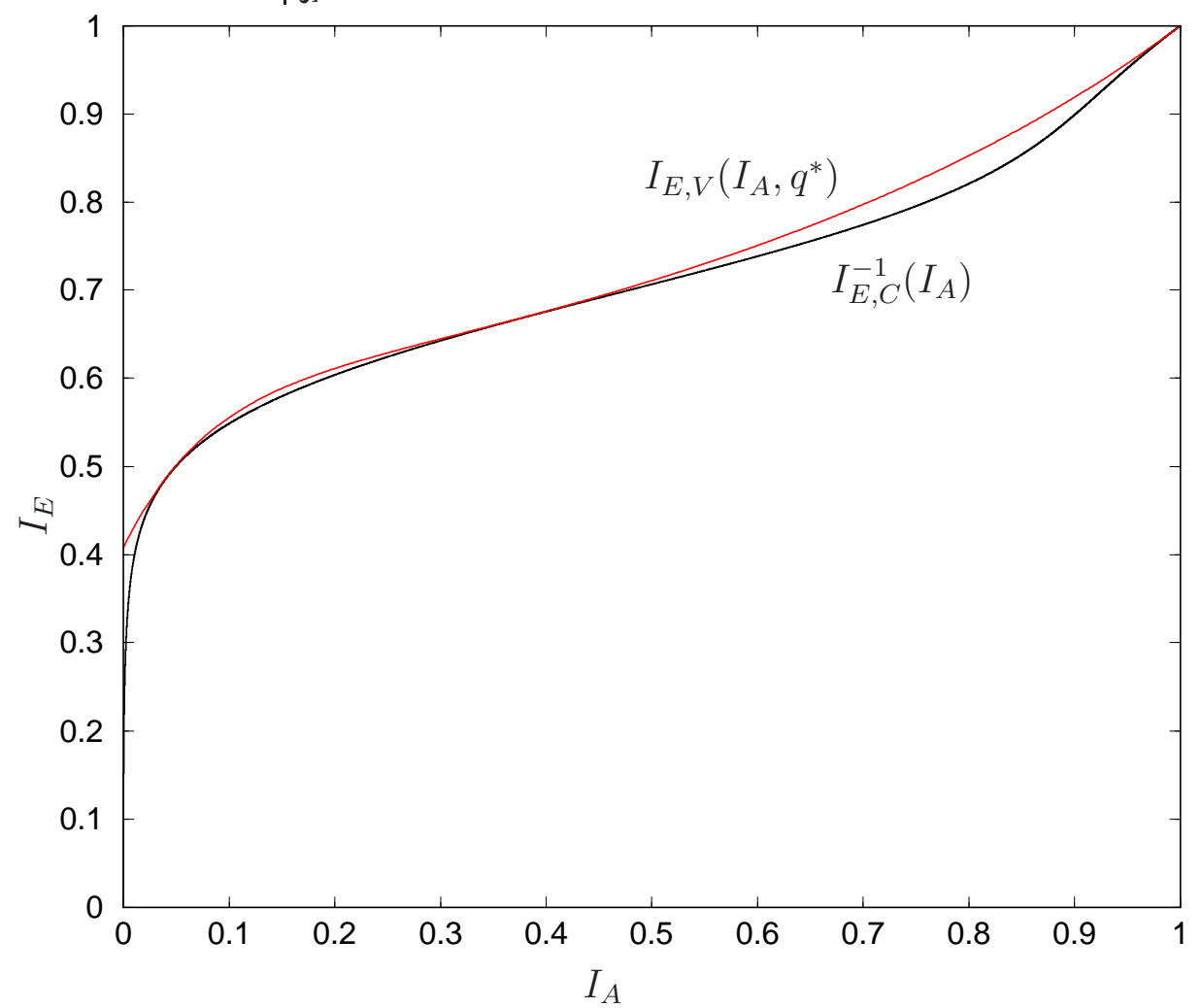

Fig. 4. EXIT chart for the D-GLDPC distribution in Table I.

binary channels," in Proc. of 2005 IEEE Global Telecommunications Conference, vol. 3, St. Louis, USA, Dec. 2005.

[18] S. ten Brink, G. Kramer, and A. Ashikhmin, "Design of low-density parity-check codes for modulation and detection," IEEE Trans. Commun., vol. 52, pp. 670-678, Apr. 2004.

[19] Y. Wang and M. Fossorier, "Doubly generalized LDPC codes over the AWGN channel," IEEE Trans. Commun., 2007, submitted.

[20] K. Price and R. Storn, "Differential evolution - a simple and efficient heuristic for global optimization over continuous spaces," J. Global Optimization, vol. 11, pp. 341-359, 1997.

[21] M. A. Shokrollahi and R. Storn, "Design of efficient erasure codes with differential evolution," in Proc. of 2001 IEEE International Symposium on Information Theory, Sorrento, Italy, June 2000. 